\title{
An imprint method for detecting leptospires in the hamster model of vaccine-mediated immunity for leptospirosis
}

Correspondence

Flávia W. C. McBride

fmcbride@ufba.br

Received 1 July 2009

Accepted 10 August 2009

\author{
Adenizar D. Chagas-Junior, ${ }^{1}$ Alan J. A. McBride, ${ }^{1}$ Daniel A. Athanazio, ${ }^{1,2}$ \\ Cláudio P. Figueira, ${ }^{1}$ Marco A. Medeiros, ${ }^{3}$ Mitermayer G. Reis, ${ }^{1}$ \\ Albert I. Ko ${ }^{1,4}$ and Flávia W. C. McBride ${ }^{1,2}$ \\ ${ }^{1}$ Gonçalo Moniz Research Centre, Oswaldo Cruz Foundation, Brazilian Ministry of Health, Salvador, \\ BA, Brazil \\ ${ }^{2}$ Department of Biointeraction, Health Sciences Institute, Federal University of Bahia, Salvador, BA, \\ Brazil \\ ${ }^{3}$ Bio-Manguinhos, Oswaldo Cruz Foundation, Brazilian Ministry of Health, Rio de Janeiro, RJ, Brazil \\ ${ }^{4}$ Division of Infectious Diseases, Weill Medical College of Cornell University, NY, USA
}

\begin{abstract}
In determining the efficacy of new vaccine candidates for leptospirosis, the primary end point is death and an important secondary end point is sterilizing immunity. However, evaluation of this end point is often hampered by the time-consuming demands and complexity of methods such as culture isolation $(\mathrm{Cl})$. In this study, we evaluated the use of an imprint (or touch preparation) method (IM) in detecting the presence of leptospires in tissues of hamsters infected with Leptospira interrogans serovar Copenhageni. In a dissemination study, compared to $\mathrm{Cl}$, the IM led to equal or improved detection of leptospires in kidney, liver, lung and blood samples collected post-infection and overall concordance was good $(\kappa=0.61)$. Furthermore, in an evaluation of hamsters immunized with a recombinant leptospiral protein-based vaccine candidate and subsequently challenged, the agreement between the $\mathrm{Cl}$ and IM was very good $(\kappa=0.84)$. These findings indicate that the IM is a rapid method for the direct observation of Leptospira spp. that can be readily applied to evaluating infection in experimental animals and determining sterilizing immunity when screening potential vaccine candidates.
\end{abstract}

\section{INTRODUCTION}

Leptospirosis is a widespread zoonosis with a global distribution, caused by pathogenic spirochaetes of the genus Leptospira (Adler \& de la Pena Moctezuma, 2009; Levett, 2001). The major impact of leptospirosis is the high rate of case fatality due to its most severe complications: Weil's disease (>10\%) (Bharti et al., 2003) and severe pulmonary haemorrhage syndrome $(>50 \%)$ (Gouveia et al., 2008; Marotto et al., 1999; Park et al., 1989). A priority in current research on leptospirosis is the development of a vaccine that is able to elicit long-term immunity and to induce cross-protection against the serovars that are of greatest importance to public health (Adler \& de la Pena Moctezuma, 2009; Koizumi \& Watanabe, 2005; McBride et al., 2005).

Abbreviations: $\mathrm{Cl}$, culture isolation; DAPI, 4',6-diamidino-2-phenylindol;

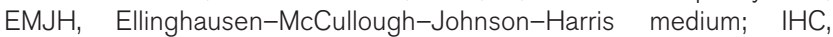
immunohistochemistry; IM, imprint method; Lig, leptospiral immunoglobulin-like; NRS, normal control rabbit antiserum; p.i., post-infection.
Several groups have reported on the use of various animal models, including mice, hamsters and gerbils, for vaccine candidate evaluation studies (Haake et al., 1999; Koizumi \& Watanabe, 2004; Palaniappan et al., 2006; Silva et al., 2007; Sonrier et al., 2000). The primary goal of any vaccine is to induce a specific immune response such that the initial infection is prevented or subsequently eliminated (Seder \& Mascola, 2003). Therefore, an important evaluation of vaccine efficacy is carriage status, as a successful vaccine should confer sterilizing immunity to the vaccinated animal. However, the gold standard protocol is culture isolation (CI), with the potential problems of contamination, incubator space requirements and delayed results (up to 8 weeks) (Palaniappan et al., 2005).

In other disease conditions, tissue imprints (or touch preparations) are commonly used for the direct detection of micro-organisms. In leishmaniasis, the direct observation of trypanosomes in tissue imprints of canine liver and spleen samples is regarded as the definitive laboratory diagnostic test (Bahamdan et al., 1996; Berger et al., 1987; 
Kar, 1995; Roscoe, 2005; Teixeira et al., 2008; Volpini et al., 2006). The technique has been applied to several infectious diseases (Jan et al., 2008; Olsen \& Stenderup, 1990; Silverman \& Gay, 1995). The aim of this study was to adapt and evaluate the imprint method (IM) for the direct observation of leptospires in samples from experimentally infected hamsters, comparing its performance against CI and immunohistochemistry (IHC).

\section{METHODS}

Leptospira strain and culture conditions. Leptospira interrogans serovar Copenhageni strain Fiocruz L1-130, isolated from a patient during an outbreak of leptospirosis in the city of Salvador, Brazil (Ko et al., 1999; Nascimento et al., 2004), was cultivated in liquid Ellinghausen-McCullough-Johnson-Harris medium (EMJH, Difco) at $29{ }^{\circ} \mathrm{C}$ (Ellinghausen \& McCullough, 1965; Johnson \& Harris, 1967) and counted in a Petroff-Hausser counting chamber (Fisher Scientific), as described by Faine et al., (1999). The strain was passaged four times in Golden Syrian hamsters (Mesocricetus auratus) and stored at $-70{ }^{\circ} \mathrm{C}$. Before use, the aliquots were thawed and passaged in liquid EMJH seven times prior to use as a low-passage isolate in the infection experiments.

Experimental infection of hamsters. Female, 9-week-old hamsters (Fiocruz) were used in leptospiral dissemination studies and in evaluating the performance of the three methods for the direct detection of Leptospira spp. (Silva et al., 2008). Groups of hamsters ( $n=5$, days 3 and 6 post-infection (p.i.); $n=15$, day 9 p.i.) were inoculated intraperitoneally with 250 leptospires in $1 \mathrm{ml}$ PBS, equivalent to $2.5 \times \mathrm{LD}_{50}$ (Silva et al., 2007), and were sacrificed at intervals ranging from 1 day to 21 days p.i. Control animals $(n=5)$ were injected intraperitoneally with $1 \mathrm{ml}$ sterile PBS. Kidney, liver, lung and blood samples were collected aseptically for evaluation. Collection of blood samples during the dissemination study was by phlebotomy of the retro-orbital venous plexus. The Ethical Committee of the Oswaldo Cruz Foundation approved all animal protocols used in this study.

Culture isolation of leptospires. CI was performed as previously described (Athanazio et al., 2008). Briefly, tissue samples (kidney, liver and lung) were homogenized in $10 \mathrm{ml} \mathrm{EMJH}$, cell debris was allowed to settle for $10 \mathrm{~min}$ and $0.5 \mathrm{ml}$ cleared homogenate was inoculated into $5 \mathrm{ml} \mathrm{EMJH}$. Blood samples (1-2 drops) were inoculated directly into $5 \mathrm{ml} \mathrm{EMJH}$, and all cultures were incubated at $29{ }^{\circ} \mathrm{C}$. The cultures were examined regularly for growth, by darkfield microscopy, for up to 8 weeks.

Immunohistochemistry. Necropsies were performed immediately upon sacrifice and kidney, lung and liver samples were stored at $-70{ }^{\circ} \mathrm{C}$ in mounting medium (Tissue-Tek, Sakura Finetek). Frozen sections $(4-5 \mu \mathrm{m})$ were thawed out onto poly-L-lysine-coated glass slides and incubated $(1 \mathrm{~h})$ with a primary polyclonal anti-leptospiral antibody (prepared by immunizing New Zealand White rabbits with a whole-cell preparation of $L$. interrogans serovar Icterohaemorrhagiae strain RGA (Athanazio et al., 2008) at a dilution of $1: 200$. After three washes in PBS, the sections were incubated $(1 \mathrm{~h})$ with goat anti-rabbit IgG-FITC conjugate (Jackson ImmunoResearch) at 1:500, and washed three times in PBS before visualization of stained organisms by fluorescence microscopy. Frozen tissue sections from non-infected hamsters were used as negative controls.

Imprint detection. Imprints were obtained by direct pressure of the cut surface of the tissue sample onto poly-L-lysine-coated glass slides.
Blood smears for imprint were prepared by placing one drop of blood onto a poly-L-lysine-coated glass slide and a smear was formed using a spreader slide. Imprint slides were dried at room temperature, fixed in acetone for $3 \mathrm{~min}$ and the smear regions demarcated with a hydrophobic barrier pen. Imprint slides were incubated $(1 \mathrm{~h})$ with a primary rabbit polyclonal anti-leptospiral antibody at a dilution of $1: 200$. After three washes in PBS, the imprints were incubated $(1 \mathrm{~h})$ with goat anti-rabbit IgG-FITC conjugate at 1:500, washed three times in PBS and dried before visualization of stained organisms by fluorescence microscopy. Tissue imprints from non-infected hamsters and normal (uninfected) rabbit serum (NRS) were used as negative controls. Counterstaining of nucleic acids or cytoplasm was achieved with $1 \mathrm{mg} \mathrm{ml}$ 4 $^{\prime}$,6-diamidino-2-phenylindole (DAPI, Sigma Aldrich) or $0.1 \%$ Evans Blue (Sigma Aldrich) at room temperature for $10 \mathrm{~min}$.

Hamster immunization and challenge studies. Recombinant LigA C-terminal protein fragments were cloned, expressed and purified as described previously (Silva et al., 2007). The subunit vaccine preparations were prepared by adsorption of the recombinant leptospiral immunoglobulin-like (Lig) proteins to aluminium hydroxide (alhydrogel) adjuvant. Immunization protocols were performed as described previously (Silva et al., 2007) with the following modifications. Briefly, female, 4-week-old hamsters were immunized by intramuscular injection on day 0 with a second immunization on day 14; control animals were injected with alhydrogel at the same intervals. The hamsters were challenged with an intraperitoneal inoculum of 250 leptospires (strain Fiocruz L1130) in $1 \mathrm{ml}$ PBS. Hamsters were monitored three times a day during the post-challenge period and euthanized when clinical signs (prostration and jaundice or pulmonary haemorrhage) of terminal disease appeared. Survivors were euthanized 21 days p.i. Kidney samples were collected from all surviving hamsters in eight independent experiments. Blood, liver and lung samples were collected from a random selection of immunized animals. The kidney samples were analysed using both CI and the IM detection methods. The IM was used to screen the remaining blood, liver and lung samples.

Statistical analysis. Statistical analysis was carried out using Epi Info, ver 6.04 (Centers for Disease Control and Prevention, USA). The chi-squared test was used to compare proportions; $P<0.05$ was considered significant. Concordance between detection methods was calculated using Cohen's Kappa (Landis \& Koch, 1977).

\section{RESULTS AND DISCUSSION}

\section{Direct observation of leptospires in a dissemination study}

The IM permitted the direct observation of leptospires by using specific anti-Leptospira whole-cell-antigen antibodies and an FITC conjugate or DAPI in kidney, liver, lung and blood samples (Fig. 1a, b). Table 1 summarizes how the three different methods performed in the detection of leptospires in kidney, liver, lung and blood samples collected from infected hamsters. Leptospires, although present, could not be detected until day 6 p.i. The IM had a higher, but not significant, rate of detection than CI on day six p.i. in the kidney ( 100 vs $60 \%$, respectively), liver (100 vs $80 \%$ ) and lung (40 vs $20 \%$ ) from infected hamsters, while the two methods exhibited a similar capacity to detect leptospires in blood samples ( 80 vs $80 \%$ ). By day 9 


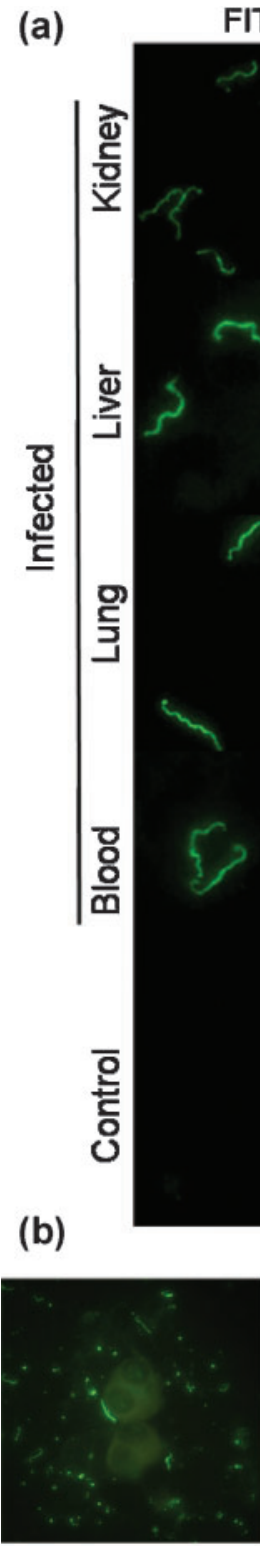

FITC

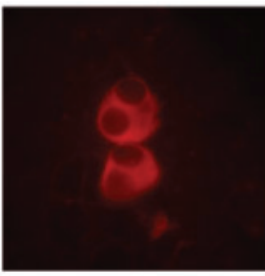

Evans Blue

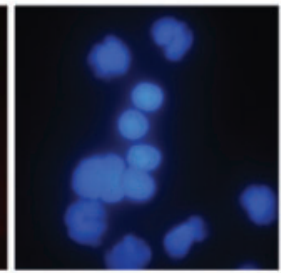

DAPI

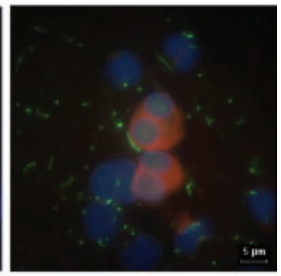

Montage
Fig. 1. Direct observation of leptospires in imprint samples. (a) Isolated leptospires were detected in kidney, liver, lung and blood imprint samples from hamsters infected with $L$. interrogans serovar Copenhageni strain Fiocruz L1-130; the negative control kidney sample was from an uninfected hamster. Normal rabbit serum (NRS) was included as a negative control. Bar, $10 \mu \mathrm{m}$. (b) Leptospires closely associated with mammalian cells were observed using FITC, cytoplasm was stained with Evans Blue, and DNA with DAPI (note the quenching of fluorescent signal from the leptospires). A montage of the superimposed images is shown. Bar, $5 \mu \mathrm{m}$. p.i., the methods detected leptospires in all 15 of the kidney samples analysed. Both the IM and IHC offered a significant improvement in detection over CI in liver (100 and $100 \%$ vs $53.3 \%$, respectively, $P<0.01$ ) and lung samples ( 80.0 and $73.3 \%$ vs $18.2 \%, P<0.01)$. The IM and CI performed similarly for blood samples (53.3 vs $46.6 \%$, respectively). When blood samples were collected daily from day 2 to day 10 p.i., leptospires were detected by day 6 p.i. and overall IM performance was equal or superior to CI. On day 9 p.i., the IM detected leptospires in $80 \%(4 / 5)$ compared to $20 \%(1 / 5)$ for CI; however, this difference was not significant (data not shown). Concordance was good for all three tests $(\kappa=0.61)$, and for CI and the IM $(\kappa=0.64)$, agreement between $\mathrm{CI}$ and IHC was moderate $(\kappa=0.49)$, while between the IM and IHC concordance was very good $(\kappa=0.96)$. When stratified according to sample type, the agreement between CI and the IM was very good in kidney $(\kappa=0.86)$ and blood samples $(\kappa=0.93)$, moderate in liver samples $(\kappa=0.50)$ and fair in lung samples $(\kappa=0.23)$.

\section{Detection of leptospires in immunized hamsters post-challenge}

Summarizing the eight experiments performed (Table 2), survival was $85.0 \%(68 / 80)$ among immunized hamsters compared to $2.5 \%(2 / 80)$ among control hamsters immunized with adjuvant only. Among the immunized hamsters which survived, cultures from eight animals could not be evaluated due to contamination. In an evaluation of sterilizing immunity, leptospires were isolated (CI) from 
Table 1. Direct detection of leptospires in a dissemination study of hamsters infected with L. interrogans strain Fiocruz L1-130

\begin{tabular}{|c|c|c|c|c|c|}
\hline \multirow[t]{2}{*}{ Days p.i. } & \multirow[t]{2}{*}{ Method* $^{*}$} & \multicolumn{4}{|c|}{ Number of positives/total (\% positive) } \\
\hline & & Kidney & Liver & Lung & Blood \\
\hline 3 & IM & $0 / 5(0)$ & $0 / 5(0)$ & $0 / 5(0)$ & $0 / 5(0)$ \\
\hline \multirow[t]{2}{*}{6} & $\mathrm{CI}$ & $3 / 5(60)$ & $4 / 5(80)$ & $1 / 5(20)$ & $4 / 5(80)$ \\
\hline & IM & $5 / 5(100)$ & $5 / 5(100)$ & $2 / 5(40)$ & $4 / 5(80)$ \\
\hline \multirow{2}{*}{9} & IHC & $15 / 15(100)$ & $15 / 15(100)$ & $11 / 15(73)$ & ND \\
\hline & IM & $15 / 15(100)$ & $15 / 15(100)$ & $12 / 15(80)$ & $8 / 15(53)$ \\
\hline \multirow[t]{3}{*}{ Uninfected controls } & $\mathrm{CI}$ & $0 / 5(0)$ & $0 / 5(0)$ & $0 / 4(0) \ddagger$ & $0 / 5(0)$ \\
\hline & IHC & $0 / 5(0)$ & $0 / 5(0)$ & $0 / 5(0)$ & ND \\
\hline & IM & $0 / 5(0)$ & $0 / 5(0)$ & $0 / 5(0)$ & $0 / 5(0)$ \\
\hline
\end{tabular}

ND, Not determined.

${ }^{\star}$ CI, culture isolation; IHC, immunohistochemistry; IM, imprint method.

$\dagger$ Four cultures were excluded due to contamination.

$\ddagger$ One culture was excluded due to contamination.

the kidneys of $23.3 \%(14 / 60)$ of surviving hamsters, whereas leptospires were detected by the IM in $26.5 \%$ $(18 / 68)$ of surviving hamsters. In a random evaluation of the IM, leptospires were detected in $42.8 \%$ (9/21) liver samples and $20.0 \%(1 / 5)$ lung samples, while none of the blood samples $(0 / 43)$ were positive. Based on the kidney samples, there was very good agreement between CI and the IM $(\kappa=0.84)$. It is of note that all samples that were CI positive were also positive by the IM.

Sterilizing immunity is an important secondary end point in vaccine candidate evaluations, particularly since it is known that commercial vaccines for animal use do not prevent leptospiruria (Adler \& de la Pena Moctezuma, 2009). The gold standard for the direct detection of leptospires in biological samples is culture isolation (Faine et al., 1999; WHO \& ILS, 2003). However, this method is not ideal due to the fastidious nature of pathogenic Leptospira spp., the need to incubate the cultures for up to 8 weeks, the considerable demand on laboratory resources when large numbers of cultures are involved, and the risk of contamination (Palaniappan et al., 2005). The main aim of this study was to adapt and evaluate the imprint method for the direct detection of leptospires in biological samples. To our knowledge, this is the first report on the application of this technique to the field of leptospirosis.

A potential limitation in the use of the IM is that although the technique identifies intact Leptospira spp. it may not discriminate between intact-viable and intact-non-viable leptospires. However, the IM only demonstrated significantly increased detection of leptospires, when compared to $\mathrm{CI}$, in the dissemination study. It is of note that in the dissemination study the presence of leptospires can be presupposed, as $100 \%$ of the infected animals died. It is known that leptospires are cleared from the blood during the first 2 weeks of infection (Faine et al., 1999; Levett, 2001); therefore if the IM was detecting intact non-viable leptospires it should have identified more positive samples by day 9 p.i. and this was not the case (Table 1). It is

Table 2. Evaluation of the IM for the detection of sterilizing immunity in the hamster model of leptospirosis

\begin{tabular}{|c|c|c|c|c|c|c|c|}
\hline \multirow[t]{3}{*}{ Survival } & Kidney & $23(14 / 60) \dagger$ & $26(18 / 68)$ & 68 & $100(2 / 2)$ & $100(2 / 2)$ & 2 \\
\hline & Liver & $\mathrm{ND}$ & $43(9 / 21)$ & & $\mathrm{ND}$ & ND & \\
\hline & Lung & $\mathrm{ND}$ & $20(1 / 5)$ & & ND & $\mathrm{ND}$ & \\
\hline Death & NA & NA & NA & 12 & NA & NA & 78 \\
\hline
\end{tabular}

ND, Not determined; NA, not applicable.

${ }^{*}$ Pooled results from eight independent experiments.

$\dagger$ Eight cultures lost to contamination. 
possible that the antigenic determinants targeted in the IM are not readily detected in intact non-viable leptospires, possibly due to loss of the outer membrane or changes in conformation or degradation of the antigens.

The leptospiral immunoglobulin-like (Lig) proteins are highly conserved, unique to pathogenic Leptospira spp. (Matsunaga et al., 2003; Cerqueira et al., 2009; McBride et al., 2009), and their use in vaccine preparations has been reported by several groups (Koizumi \& Watanabe, 2004; Palaniappan et al., 2006). We reported that hamsters immunized with a LigA C-terminal fragment in Freund's adjuvant were significantly protected (67-100\%), but that all survivors exhibited evidence of renal colonization (Silva et al., 2007). In addition, LigA vaccine formulations were reported to confer $75-100 \%$ protection, with sterilizing immunity ranging from 25 to $90 \%$ (Faisal et al., 2008, 2009; Palaniappan et al., 2006). A vaccine preparation of LigB and alhydrogel induced significant protection in hamsters (54-83\%) but did not confer sterilizing immunity (Yan et al., 2009). In C3H/HeJ mice, immunization with Lig proteins elicited significant protection (100\%), but not sterilizing immunity (Koizumi \& Watanabe, 2004). Haake et al., (1999) found that hamsters immunized with recombinant OmpL1 and LipL41 were significantly protected (71-100\%) and sterilizing immunity was $100 \%$. Such variability in sterilizing immunity may be associated with the virulence of the challenge strain, as the more virulent challenge strains resulted in kidney colonization (Silva et al., 2007; Faisal et al., 2008, 2009; Yan et al., 2009); however, this was not always the case (Haake et al., 1999). Yet another factor could be the poor reproducibility of CI, highlighting the urgent need for an improved standardized method. Among the eight cultures that were contaminated during CI, three were imprint positive. This suggests that the imprint technique could provide more reproducible results than $\mathrm{CI}$ in evaluating sterilizing immunity.

The IM proved to be a robust and sensitive method for the direct observation of intact leptospires in blood and tissue samples. Although the technique was equal to IHC in terms of detection, the IM had advantages in terms of its relative simplicity and reduced time to result. More importantly, the IM performed equally to $\mathrm{CI}$ in evaluating sterilizing immunity (Table 2) in the hamster model of leptospirosis. The use of the IM could also be applied to the direct examination of urine, as this is traditionally evaluated by darkfield microscopy and is unreliable (WHO \& ILS, 2003). This could lead to the application of the IM in field evaluations of leptospiral carriage among natural reservoirs; this is currently being evaluated.

In conclusion, we report the successful adaptation and evaluation of the imprint method for the direct detection of pathogenic Leptospira spp. in blood and tissue samples collected from an experimental animal model. Furthermore, the technique was readily applied to the evaluation of sterilizing immunity in vaccine-mediated immunity studies. We therefore propose that the imprint technique should be considered as a complementary method for the detection of leptospires in animal models of leptospirosis.

\section{ACKNOWLEDGEMENTS}

This work was supported by the Bahia State Foundation for Research Support (FAPESB), grants 5108/2005, PES-0092/2008 and 54663; the Oswaldo Cruz Foundation; the Brazilian National Research Council, grant 554788/2006; and the National Institutes of Health, USA, grants R01 AI052473 and D43 TW-00919. The authors are grateful to Cleiton Silva Santos and Jefferson Alves for technical assistance in the experimental procedures.

\section{REFERENCES}

Adler, B. \& de la Pena Moctezuma, A. (2009). Leptospira and leptospirosis. Vet Microbiol 2009 Mar 13 [Epub ahead of print].

Athanazio, D. A., Silva, E. F., Santos, C. S., Rocha, G. M., VannierSantos, M. A., McBride, A. J., Ko, A. I. \& Reis, M. G. (2008). Rattus norvegicus as a model for persistent renal colonization by pathogenic Leptospira interrogans. Acta Trop 105, 176-180.

Bahamdan, K. A., Khan, A. R., Tallab, T. M. \& Mourad, M. M. (1996). Value of touch preparations (imprints) for diagnosis of cutaneous leishmaniasis. Int J Dermatol 35, 558-560.

Berger, R. S., Perez-Figaredo, R. A. \& Spielvogel, R. L. (1987). Leishmaniasis: the touch preparation as a rapid means of diagnosis. J Am Acad Dermatol 16, 1096-1105.

Bharti, A. R., Nally, J. E., Ricaldi, J. N., Matthias, M. A., Diaz, M. M., Lovett, M. A., Levett, P. N., Gilman, R. H., Willig, M. R. \& other authors (2003). Leptospirosis: a zoonotic disease of global importance. Lancet Infect Dis 3, 757-771.

Cerqueira, G. M., McBride, A. J., Picardeau, M., Ribeiro, S. G., Moreira, A. N., Morel, V., Reis, M. G., Ko, A. I. \& Dellagostin, O. A. (2009). Distribution of the leptospiral immunoglobulin-like (Lig) genes in pathogenic Leptospira spp. and application of ligB to typing leptospiral isolates. J Med Microbiol 58, 1173-1181.

Ellinghausen, H. C., Jr \& McCullough, W. G. (1965). Nutrition of Leptospira pomona and growth of 13 other serotypes: fractionation of oleic albumin complex and a medium of bovine albumin and polysorbate 80. Am J Vet Res 26, 45-51.

Faine, S. B., Adler, B., Bolin, C. \& Perolat, P. (1999). Leptospira and Leptospirosis, 2nd edn. Melbourne, Australia: MediSci.

Faisal, S. M., Yan, W., Chen, C. S., Palaniappan, R. U., McDonough, S. P. \& Chang, Y. F. (2008). Evaluation of protective immunity of Leptospira immunoglobulin like protein A (LigA) DNA vaccine against challenge in hamsters. Vaccine 26, 277-287.

Faisal, S. M., Yan, W., McDonough, S. P. \& Chang, Y. F. (2009). Leptospira immunoglobulin-like protein A variable region (LigAvar) incorporated in liposomes and PLGA microspheres produces a robust immune response correlating to protective immunity. Vaccine 27, 378-387.

Gouveia, E. L., Metcalfe, J., de Carvalho, A. L. F., Aires, T. S., Villasboas-Bisneto, J. C., Queirroz, A., Santos, A. C., Salgado, K., Reis, M. G. \& Ko, A. I. (2008). Leptospirosis-associated severe pulmonary hemorrhage syndrome, Salvador, Brazil. Emerg Infect Dis 14, 505-508.

Haake, D. A., Mazel, M. K., McCoy, A. M., Milward, F., Chao, G., Matsunaga, J. \& Wagar, E. A. (1999). Leptospiral outer membrane proteins OmpL1 and LipL41 exhibit synergistic immunoprotection. Infect Immun 67, 6572-6582. 
Jan, I. S., Chung, P. F., Wang, J. Y., Weng, M. H., Hung, C. C. \& Lee, L. N. (2008). Cytological diagnosis of Penicillium marneffei infection. $J$ Formos Med Assoc 107, 443-447.

Johnson, R. C. \& Harris, V. G. (1967). Differentiation of pathogenic and saprophytic leptospires. I. Growth at low temperatures.J Bacteriol 94, 27-31.

Kar, K. (1995). Serodiagnosis of leishmaniasis. Crit Rev Microbiol 21, 123-152.

Ko, A. I., Galvao Reis, M., Ribeiro Dourado, C. M., Johnson, W. D., Jr \& Riley, L. W. (1999). Urban epidemic of severe leptospirosis in Brazil. Salvador Leptospirosis Study Group. Lancet 354, 820-825.

Koizumi, N. \& Watanabe, H. (2004). Leptospiral immunoglobulin-like proteins elicit protective immunity. Vaccine 22, 1545-1552.

Koizumi, N. \& Watanabe, H. (2005). Leptospirosis vaccines: past, present, and future. J Postgrad Med 51, 210-214.

Landis, J. R. \& Koch, G. G. (1977). The measurement of observer agreement for categorical data. Biometrics 33, 159-174.

Levett, P. N. (2001). Leptospirosis. Clin Microbiol Rev 14, 296-326.

Marotto, P. C., Nascimento, C. M., Eluf-Neto, J., Marotto, M. S., Andrade, L., Sztajnbok, J. \& Seguro, A. C. (1999). Acute lung injury in leptospirosis: clinical and laboratory features, outcome, and factors associated with mortality. Clin Infect Dis 29, 1561-1563.

Matsunaga, J., Barocchi, M. A., Croda, J., Young, T. A., Sanchez, Y., Siqueira, I., Bolin, C. A., Reis, M. G., Riley, L. W. \& other authors (2003). Pathogenic Leptospira species express surface-exposed proteins belonging to the bacterial immunoglobulin superfamily. Mol Microbiol 49, 929-945.

McBride, A. J., Athanazio, D. A., Reis, M. G. \& Ko, A. I. (2005). Leptospirosis. Curr Opin Infect Dis 18, 376-386.

McBride, A. J., Cerqueira, G. M., Suchard, M. A., Moreira, A. N., Zuerner, R. L., Reis, M. G., Haake, D. A., Ko, A. I. \& Dellagostin, O. A. (2009). Genetic diversity of the leptospiral immunoglobulin-like (Lig) genes in pathogenic Leptospira spp. Infect Genet Evol 9, 196-205.

Nascimento, A. L., Ko, A. I., Martins, E. A., Monteiro-Vitorello, C. B., Ho, P. L., Haake, D. A., Verjovski-Almeida, S., Hartskeerl, R. A., Marques, M. V. \& other authors (2004). Comparative genomics of two Leptospira interrogans serovars reveals novel insights into physiology and pathogenesis. J Bacteriol 186, 2164-2172.

Olsen, I. \& Stenderup, A. (1990). Clinical-mycologic diagnosis of oral yeast infections. Acta Odontol Scand 48, 11-18.

Palaniappan, R. U., Chang, Y. F., Chang, C. F., Pan, M. J., Yang, C. W., Harpending, P., McDonough, S. P., Dubovi, E., Divers, T. \& other authors (2005). Evaluation of Lig-based conventional and real time PCR for the detection of pathogenic leptospires. Mol Cell Probes 19, $111-117$.
Palaniappan, R. U., McDonough, S. P., Divers, T. J., Chen, C. S., Pan, M. J., Matsumoto, M. \& Chang, Y. F. (2006). Immunoprotection of recombinant leptospiral immunoglobulin-like protein A against Leptospira interrogans serovar Pomona infection. Infect Immun 74, 1745-1750.

Park, S. K., Lee, S. H., Rhee, Y. K., Kang, S. K., Kim, K. J., Kim, M. C., Kim, K. W. \& Chang, W. H. (1989). Leptospirosis in Chonbuk Province of Korea in 1987: a study of 93 patients. Am J Trop Med Hyg 41, 345351.

Roscoe, M. (2005). Leishmaniasis: early diagnosis is key. JAAPA $\mathbf{1 8}$, 47-50, 53-44.

Seder, R. A. \& Mascola, J. R. (2003). Basic immunology of vaccine development. In The Vaccine Book, pp. 51-72. Edited by B. R. Bloom \& P.-H. Lambert. San Diego: Academic Press.

Silva, E. F., Medeiros, M. A., McBride, A. J., Matsunaga, J., Esteves, G. S., Ramos, J. G., Santos, C. S., Croda, J., Homma, A. \& other authors (2007). The terminal portion of leptospiral immunoglobulin-like protein LigA confers protective immunity against lethal infection in the hamster model of leptospirosis. Vaccine 25, 6277-6286.

Silva, E. F., Santos, C. S., Athanazio, D. A., Seyffert, N., Seixas, F. K., Cerqueira, G. M., Fagundes, M. Q., Brod, C. S., Reis, M. G. \& other authors (2008). Characterization of virulence of Leptospira isolates in a hamster model. Vaccine 26, 3892-3896.

Silverman, J. F. \& Gay, R. M. (1995). Fine-needle aspiration and surgical pathology of infectious lesions. Morphologic features and the role of the clinical microbiology laboratory for rapid diagnosis. Clin Lab Med 15, 251-278.

Sonrier, C., Branger, C., Michel, V., Ruvoen-Clouet, N., Ganiere, J. P. \& Andre-Fontaine, G. (2000). Evidence of cross-protection within Leptospira interrogans in an experimental model. Vaccine 19, 86-94.

Teixeira, A. C., Paes, M. G., Guerra Jde, O., Prata, A. \& Silva-Vergara, M. L. (2008). Failure of both azithromycin and antimony to treat cutaneous leishmaniasis in Manaus, AM, Brazil. Rev Inst Med Trop Sao Paulo 50, 157-160.

Volpini, A. C., Marques, M. J., Lopes dos Santos, S., MachadoCoelho, G. L., Mayrink, W. \& Romanha, A. J. (2006). Leishmania identification by PCR of Giemsa-stained lesion imprint slides stored for up to 36 years. Clin Microbiol Infect 12, 815-818.

WHO \& ILS (2003). Human Leptospirosis: Guidance for Diagnosis, Surveillance and Control. Malta: World Health Organization.

Yan, W., Faisal, S. M., McDonough, S. P., Divers, T. J., Barr, S. C., Chang, C. F., Pan, M. J. \& Chang, Y. F. (2009). Immunogenicity and protective efficacy of recombinant Leptospira immunoglobulin-like protein B (rLigB) in a hamster challenge model. Microbes Infect 11, 230-237. 